\title{
SMOOTHING PROPERTIES OF THE DISCRETE FRACTIONAL MAXIMAL OPERATOR ON BESOV AND TRIEBEL-LIZORKIN SPACES
}

\author{
Toni Heikkinen And Heli Tuominen
}

\begin{abstract}
Motivated by the results of Korry, and Kinnunen and Saksman, we study the behaviour of the discrete fractional maximal operator on fractional Hajłasz spaces, Hajłasz-Besov, and Hajłasz-Triebel-Lizorkin spaces on metric measure spaces. We show that the discrete fractional maximal operator maps these spaces to the spaces of the same type with higher smoothness. Our results extend and unify aforementioned results. We present our results in a general setting, but they are new already in the Euclidean case.
\end{abstract}

2010 Mathematics Subject Classification: 42B25, 46E35.

Key words: Besov space, fractional maximal function, fractional Sobolev space, Triebel-Lizorkin space, metric measure space.

\section{Introduction}

Maximal functions are standard tools in harmonic analysis. They are usually used to estimate absolute size, but recently there has been interest in studying their regularity properties, see $[\mathbf{1}],[\mathbf{2}],[\mathbf{3}],[\mathbf{1 2}]$, $[\mathbf{1 3}],[\mathbf{1 4}],[\mathbf{1 6}],[\mathbf{1 8}],[\mathbf{1 9}],[\mathbf{2 0}],[\mathbf{2 2}],[\mathbf{2 3}],[\mathbf{2 4}],[\mathbf{2 6}],[\mathbf{2 7}]$, and $[\mathbf{2 9}]$. A starting point was the article [18] by Kinnunen, where he observed that the Hardy-Littlewood maximal operator is bounded on $W^{1, p}\left(\mathbb{R}^{n}\right)$ for $1<p \leq \infty$. In [23] and [24] Korry extended this result by showing that the maximal operator preserves also fractional Sobolev spaces as well as Besov and Triebel-Lizorkin spaces. Another kind of extension was given in $[\mathbf{2 1}]$, where Kinnunen and Saksman showed that the fractional maximal operator $\mathcal{M}_{\alpha}$, defined by

$$
\mathcal{M}_{\alpha} u(x)=\sup _{r>0} \frac{r^{\alpha}}{|B(x, r)|} \int_{B(x, r)}|u(y)| d y,
$$

is bounded from $W^{1, p}\left(\mathbb{R}^{n}\right)$ to $W^{1, p^{*}}\left(\mathbb{R}^{n}\right)$, where $p^{*}=n p /(n-\alpha p)$, and from $L^{p}\left(\mathbb{R}^{n}\right)$ to $\dot{W}^{1, q}\left(\mathbb{R}^{n}\right)$, where $q=n p /(n-(\alpha-1) p)$, and $\dot{W}^{1, q}\left(\mathbb{R}^{n}\right)$ is the homogenous Sobolev space. These results indicate that $\mathcal{M}_{\alpha}$ has similar smoothing properties as the Riesz potential. 
It is natural to ask whether these results can be seen as special cases of the behaviour of the fractional maximal operator on Besov and Triebel-Lizorkin spaces. In this paper, we show that this is indeed the case, and that all these results can be obtained by the same rather simple method. Instead of the standard fractional maximal operator, we consider its variant, the so-called discrete fractional maximal operator $\mathcal{M}_{\alpha}^{*}$. This allows us to present our results in a setting of doubling metric measure spaces. In this generality, the standard fractional maximal operator behaves quite badly. Indeed, one can construct spaces, where the fractional maximal function of a Lipschitz function fails to be continuous, see $[\mathbf{3}]$ and $[\mathbf{1 6}]$. Since $\mathcal{M}_{\alpha}^{*}$ and $\mathcal{M}_{\alpha}$ are comparable, for many practical purposes it does not matter which one we choose. The discrete fractional maximal operator was introduced in [19] and further studied in [22], [1], and $[\mathbf{1 4}]$.

Among the many possible definitions of Besov and Triebel-Lizorkin spaces, the most suitable for our purposes is the one based on Hajłasz type pointwise inequalities. This approach, introduced by Koskela, Yang, and Zhou in [25], provides a new point of view to the classical Besov and Triebel-Lizorkin spaces. On the other hand, it allows these spaces to be defined in the setting of metric measure spaces.

By employing this definition, we can prove very general results using only simple "telescoping" arguments and Poincaré type inequalities. As special cases, we obtain versions of the results of Kinnunen and Saksman as well as those of Korry, see Remark 3.5, and Theorems 4.7 and 4.8. We prove our results in doubling metric measure spaces but they are new even in Euclidean spaces. Our main results (Theorems 4.5 and 4.6) imply that if $\alpha \geq 0$ and $0<s+\alpha<1$, then $\mathcal{M}_{\alpha}^{*}$ is bounded from $\dot{F}_{p, q}^{s}\left(\mathbb{R}^{n}\right)$ to $\dot{F}_{p, q}^{s+\alpha}\left(\mathbb{R}^{n}\right)$ for $n /(n+s)<p, q<\infty$ and from $\dot{B}_{p, q}^{s}\left(\mathbb{R}^{n}\right)$ to $\dot{B}_{p, q}^{s+\alpha}\left(\mathbb{R}^{n}\right)$ for $n /(n+s)<p<\infty, 0<q<\infty$, see Section 4 for the definition of Triebel-Lizorkin and Besov spaces.

\section{Preliminaries and notation}

We assume that $X=(X, d, \mu)$ is a metric measure space equipped with a metric $d$ and a Borel regular outer measure $\mu$, which satisfies $0<\mu(U)<\infty$ whenever $U$ is nonempty, open, and bounded. We assume that the measure is doubling, that is, there exists a fixed constant $c_{d}>0$, called the doubling constant, such that

$$
\mu(B(x, 2 r)) \leq c_{d} \mu(B(x, r))
$$

for every ball $B(x, r)=\{y \in X: d(y, x)<r\}$. 
The doubling condition implies that

$$
\frac{\mu(B(y, r))}{\mu(B(x, R))} \geq C\left(\frac{r}{R}\right)^{Q}
$$

for every $0<r \leq R$ and $y \in B(x, R)$ for some $C>0$ and $Q>1$ that only depend on $c_{D}$. In fact, we may take $Q=\log _{2} c_{d}$.

For the boundedness of the fractional maximal operator in $L^{p}$, we have to assume, in Theorems 2.1 and $3.4 \mathrm{~b}$ ), that the measure $\mu$ satisfies the lower bound condition

$$
\mu(B(x, r)) \geq c_{l} r^{Q}
$$

with some constant $c_{l}>0$ for all $x \in X$ and $r>0$.

By saying that a function $u: X \rightarrow[-\infty, \infty]$ is locally integrable, we mean that is integrable on balls. Similarly, the class of functions that belong to $L^{p}(B), p>0$, in all balls $B$, is denoted by $L_{\text {loc }}^{p}(X)$. The integral average of a locally integrable function $u$ over a ball $B$ is

$$
u_{B}=f_{B} u d \mu=\frac{1}{\mu(B)} \int_{B} u d \mu .
$$

The fractional maximal function. Let $\alpha \geq 0$. The fractional maximal function of a locally integrable function $u$ is

$$
\mathcal{M}_{\alpha} u(x)=\sup _{r>0} r^{\alpha} f_{B(x, r)}|u| d \mu .
$$

For $\alpha=0$, we have the usual Hardy-Littlewood maximal function

$$
\mathcal{M} u(x)=\sup _{r>0} f_{B(x, r)}|u| d \mu .
$$

The Hardy-Littlewood maximal operator $\mathcal{M}$ is bounded in $L^{p}$ in metric measure spaces with a doubling measure when $1<p \leq \infty$, see [11, Theorem 14.13]. This, together with the Hölder inequality implies the following Sobolev type inequality for the fractional maximal operator. For the proof, see $[\mathbf{4}],[\mathbf{6}]$ or $[\mathbf{1 4}$, Theorem 3.2].

Theorem 2.1. Assume that measure lower bound condition (2.3) holds. If $p>1$ and $0<\alpha<Q / p$, then there is a constant $C>0$, depending only on the doubling constant, constant in the measure lower bound, $p$ and $\alpha$, such that

$$
\left\|\mathcal{M}_{\alpha} u\right\|_{L^{p^{*}}(X)} \leq C\|u\|_{L^{p}(X)},
$$

for every $u \in L^{p}(X)$ with $p^{*}=Q p /(Q-\alpha p)$. 
The discrete fractional maximal function. We begin the construction of the discrete maximal function with a covering of the space. Let $r>0$. Since the measure is doubling, there are balls $B\left(x_{i}, r\right)$, $i=1,2, \ldots$, such that

$$
X=\bigcup_{i} B\left(x_{i}, r\right) \quad \text { and } \quad \sum_{i} \chi_{B\left(x_{i}, 6 r\right)} \leq N<\infty
$$

where $\chi_{B\left(x_{i}, 6 r\right)}$ is the characteristic function of the ball $B\left(x_{i}, 6 r\right)$. This means that the dilated balls $B\left(x_{i}, 6 r\right), i=1,2, \ldots$, are of bounded overlap. The constant $N$ depends only on the doubling constant and, in particular, it is independent of $r$.

Then we construct a partition of unity subordinate to the covering $B\left(x_{i}, r\right), i=1,2, \ldots$, of $X$. Indeed, there is a family of functions $\varphi_{i}$, $i=1,2, \ldots$, such that for each $i, 0 \leq \varphi_{i} \leq 1, \varphi_{i}=0$ in $X \backslash B\left(x_{i}, 6 r\right)$, $\varphi_{i} \geq \nu$ in $B\left(x_{i}, 3 r\right), \varphi_{i}$ is Lipschitz with constant $L / r$ with $\nu$ and $L$ depending only on the doubling constant, and

$$
\sum_{i} \varphi_{i}(x)=1
$$

for every $x \in X$.

The discrete convolution of a locally integrable function $u$ at the scale $3 r$ is

$$
u_{r}(x)=\sum_{i} \varphi_{i}(x) u_{B\left(x_{i}, 3 r\right)}
$$

for every $x \in X$, and we write $u_{r}^{\alpha}=r^{\alpha} u_{r}$.

Let $r_{j}, j=1,2, \ldots$, be an enumeration of the positive rationals and let balls $B\left(x_{i}, r_{j}\right), i=1,2, \ldots$, be a covering of $X$ as above. The discrete fractional maximal function of $u$ in $X$ is $\mathcal{M}_{\alpha}^{*} u$,

$$
\mathcal{M}_{\alpha}^{*} u(x)=\sup _{j}|u|_{r_{j}}^{\alpha}(x)
$$

for every $x \in X$. For $\alpha=0$, we obtain the Hardy-Littlewood type discrete maximal function $\mathcal{M}^{*} u$ studied in [19], [22], and [1]. Observe that the construction depends on the choice of the coverings, but we will derive estimates that are independent of the chosen coverings.

The discrete fractional maximal function is easily seen to be comparable to the standard fractional maximal function, that is,

$$
C^{-1} \mathcal{M}_{\alpha} u \leq \mathcal{M}_{\alpha}^{*} u \leq C \mathcal{M}_{\alpha} u,
$$

where $C>1$ depends only on the doubling constant, see [14, Lemma 5.1]. 
Remark 2.2. If $u$ is only locally integrable, then $\mathcal{M}_{\alpha}^{*} u$ may well be identically infinite. However, if $\mathcal{M}_{\alpha}^{*} u\left(x_{0}\right)<\infty$ for some $x_{0} \in X$, then $\mathcal{M}_{\alpha}^{*} u(x)<\infty$ for almost every $x$.

By (2.5), it suffices to show that if $\mathcal{M}_{\alpha} u\left(x_{0}\right)<\infty$ for some $x_{0} \in X$, then $\mathcal{M}_{\alpha} u(x)<\infty$ for almost every $x$. This follows from the estimate

$$
r^{\alpha} f_{B(x, r)}|u| d \mu \leq \frac{\mu\left(B\left(x_{0}, r+d\left(x, x_{0}\right)\right)\right)}{\mu(B(x, r))} \mathcal{M}_{\alpha} u\left(x_{0}\right)
$$

combined with the doubling condition and the fact that

$$
\lim _{r \rightarrow 0} r^{\alpha} f_{B(x, r)}|u| d \mu<\infty,
$$

whenever $x$ is a Lebesgue point of $u$.

Throughout the paper, $C$ will denote a positive constant whose value is not necessarily the same at each occurrence. In the results of Sections 3 and 4 , the constants $C$ do not depend on the functions, gradients or the coverings used in the definition of the discrete fractional maximal function. They may depend on the doubling constant, the parameters of the space and constants of lemmas that are used in the proof, for example on constants of Poincaré type inequalities.

\section{Fractional Hajłasz spaces}

In this section, we study smoothing properties of the discrete fractional maximal operator on fractional Hajłasz spaces, fractional Sobolev spaces in metric spaces defined using a pointwise definition.

Let $u: X \rightarrow[-\infty, \infty]$ be a measurable function and let $0 \leq s<\infty$. A nonnegative measurable function $g$ is an $s$-Hajłasz gradient of $u$ if there exists $E \subset X$ with $\mu(E)=0$ such that for all $x, y \in X \backslash E$,

$$
|u(x)-u(y)| \leq d(x, y)^{s}(g(x)+g(y)) .
$$

The collection of all $s$-Hajłasz gradients of $u$ is denoted by $\mathcal{D}^{s}(u)$.

Let $0 \leq s<\infty$ and $0<p \leq \infty$. A homogeneous Hajłasz space $\dot{M}^{s, p}(X)$ consists of measurable functions $u$ such that

$$
\|u\|_{\dot{M}^{s, p}(X)}=\inf _{g \in \mathcal{D}^{s}(u)}\|g\|_{L^{p}(X)}
$$

is finite. The Hajłasz space $M^{s, p}(X)$ is $\dot{M}^{s, p}(X) \cap L^{p}(X)$ equipped with the norm (quasinorm when $0<p<1$ )

$$
\|u\|_{M^{s, p}(X)}=\|u\|_{L^{p}(X)}+\|u\|_{\dot{M}^{s, p}(X)} .
$$

The space $M^{1, p}(X)$, a counterpart of a Sobolev space in a metric measure space, was introduced in $[\mathbf{9}]$, see also for example [10]. The fractional 
spaces $M^{s, p}(X)$ were introduced in [31] and studied for example in [17] and [15]. Notice that $M^{0, p}(X)=L^{p}(X)$.

The pointwise definition of the Hajłasz spaces implies the validity of Sobolev-Poincaré type inequalities without an assumption that the space admits a weak Poincaré inequality.

The following lemma from [7, Lemma 2.2] is a generalisation of $[\mathbf{1 0}$, Theorem 8.7].

Lemma 3.1. Let $0<s<\infty$ and let $0<p<Q / s$. There exists a constant $C>0$ such that for all measurable functions $u$ with $g \in \mathcal{D}^{s}(u)$, all $x \in X$, and $r>0$,

$$
\inf _{c \in \mathbb{R}}\left(f_{B(x, r)}|u(y)-c|^{p^{*}(s)} d \mu(y)\right)^{1 / p^{*}(s)} \leq C r^{s}\left(f_{B(x, 2 r)} g^{p} d \mu\right)^{1 / p}
$$

where $p^{*}(s)=Q p /(Q-s p)$.

Since $(Q /(Q+s))^{*}(s)=1,(3.2)$ implies that

$$
\inf _{c \in \mathbb{R}} f_{B(x, r)}|u-c| d \mu \leq C r^{s}\left(f_{B(x, 2 r)} g^{Q /(Q+s)} d \mu\right)^{(Q+s) / Q} .
$$

Hence, if $p \geq Q /(Q+s)$ and $g \in \mathcal{D}^{s}(u) \cap L_{\text {loc }}^{p}(X)$, then $u$ is locally integrable and

$$
f_{B(x, r)}\left|u-u_{B(x, r)}\right| d \mu \leq C r^{s}\left(f_{B(x, 2 r)} g^{p} d \mu\right)^{1 / p} .
$$

Note that, by the integrability of $u$, the left sides of (3.3) and (3.4) are comparable. Inequality (3.4) holds also when $s=0$ and $p \geq 1$. This follows easily from (3.1).

In the proof of Theorem 3.3, we use the following simple result.

Lemma 3.2. If $u_{i}: X \rightarrow[-\infty, \infty], i \in \mathbb{N}$, are measurable functions with a common $s$-Hajtasz gradient $g$ and $u=\sup _{i} u_{i}$ is finite almost everywhere, then $g$ is an $s$-Hajtasz gradient of $u$.

Proof: As a supremum of countable many measurable functions, $u$ is measurable.

Let $x, y \in X$ such that $u(x)<\infty$ and $u(y)<\infty$. We may assume that $u(x) \geq u(y)$. Let $\varepsilon>0$. There exists $i$ such that $u(x) \leq u_{i}(x)+\varepsilon$. Since $u(y) \geq u_{i}(y)$ and $g$ is an $s$-Hajłasz gradient of $u_{i}$, we have

$$
u(x)-u(y) \leq u_{i}(x)-u_{i}(y)+\varepsilon \leq d(x, y)^{s}(g(x)+g(y))+\varepsilon .
$$

The claim follows by letting $\varepsilon \rightarrow 0$. 
Theorem 3.3. Let $s \geq 0, \alpha \geq 0$ and let $t \geq Q /(Q+s)$.

a) If $0<s+\alpha \leq 1$, then there exists a constant $C>0$ such that

$$
\tilde{g}=C\left(\mathcal{M} g^{t}\right)^{1 / t}
$$

is an $(s+\alpha)$-Hajtasz gradient of $\mathcal{M}_{\alpha}^{*} u$ whenever $u: X \rightarrow[-\infty, \infty]$ is a measurable function such that $\mathcal{M}_{\alpha}^{*} u \not \equiv \infty$ and $g \in L_{\mathrm{loc}}^{t}(X)$ is an $s$-Hajtasz gradient of $u$.

b) If $s+\alpha>1$, then there exists a constant $C>0$ such that

$$
\tilde{g}=C\left(\mathcal{M}_{t(s+\alpha-1)} g^{t}\right)^{1 / t}
$$

is a 1-Hajtasz gradient of $\mathcal{M}_{\alpha}^{*} u$ whenever $u: X \rightarrow[-\infty, \infty]$ is a measurable function such that $\mathcal{M}_{\alpha}^{*} u \not \equiv \infty$ and $g \in L_{\mathrm{loc}}^{t}(X)$ is an $s$-Hajtasz gradient of $u$.

Proof: Since $\mathcal{M}_{\alpha}^{*} u=\mathcal{M}_{\alpha}^{*}|u|$ and since every $s$-Hajłasz gradient of $u$ is an $s$-Hajłasz gradient of $|u|$, we may assume that $u \geq 0$.

We begin by proving the claims for $u_{r}^{\alpha}$. Let $r>0$, let $g \in \mathcal{D}^{s}(u)$ and let $x, y \in X$.

Assume first that $r \geq d(x, y)$. Let $I_{x y}$ be a set of indices $i$ for which $x$ or $y$ belongs to $B\left(x_{i}, 6 r\right)$. Then, for each $i \in I_{x y}, B\left(x_{i}, 3 r\right) \subset B(x, 10 r) \subset$ $B\left(x_{i}, 17 r\right)$. This together with the doubling condition, the properties of the functions $\varphi_{i}$, the fact that there are bounded number of indices in $I_{x y}$ and Poincaré inequality (3.4) implies that

$$
\begin{aligned}
\left|u_{r}^{\alpha}(x)-u_{r}^{\alpha}(y)\right| & \leq r^{\alpha} \sum_{i \in I_{x y}}\left|\varphi_{i}(x)-\varphi_{i}(y)\right|\left|u_{B\left(x_{i}, 3 r\right)}-u_{B(x, 10 r)}\right| \\
& \leq C r^{\alpha-1} d(x, y) \sum_{i \in I_{x y}} f_{B(x, 10 r)}\left|u-u_{B\left(x_{i}, 3 r\right)}\right| d \mu \\
& \leq C r^{\alpha-1} d(x, y) f_{B(x, 10 r)}\left|u-u_{B(x, 10 r)}\right| d \mu \\
& \leq C r^{s+\alpha-1} d(x, y)\left(f_{B(x, 20 r)} g^{t} d \mu\right)^{1 / t} .
\end{aligned}
$$

If $0<s+\alpha \leq 1$, then by (3.5) and the assumption $r \geq d(x, y)$, we have that

$$
\left|u_{r}^{\alpha}(x)-u_{r}^{\alpha}(y)\right| \leq C d(x, y)^{s+\alpha}\left(\mathcal{M} g^{t}(x)\right)^{1 / t} .
$$

If $s+\alpha>1$, then by $(3.5)$,

$$
\left|u_{r}^{\alpha}(x)-u_{r}^{\alpha}(y)\right| \leq C d(x, y)\left(\mathcal{M}_{t(s+\alpha-1)} g^{t}(x)\right)^{1 / t} .
$$


This shows that Hajłasz gradient inequality (3.1) with desired exponent holds when $r \geq d(x, y)$.

Assume then that $r<d(x, y)$. Let $R=d(x, y)$. Then $B(y, r) \subset$ $B(x, 2 R)$ and

$$
\begin{aligned}
\left|u_{r}^{\alpha}(x)-u_{r}^{\alpha}(y)\right| \leq r^{\alpha} & \left(\sum_{i \in I_{x}} \varphi_{i}(x)\left|u_{B\left(x_{i}, 3 r\right)}-u_{B(x, 9 R)}\right|\right. \\
& \left.+\sum_{i \in I_{y}} \varphi_{i}(y)\left|u_{B\left(x_{i}, 3 r\right)}-u_{B(x, 9 R)}\right|\right),
\end{aligned}
$$

where $I_{x}$ is a set of indices $i$ for which $x$ belongs to $B\left(x_{i}, 6 r\right)$ and $I_{y}$ the corresponding set for $y$. Let $k \in \mathbb{N}$ be the smallest integer such that $2^{k} r \geq R$.

Assume first that $0<s+\alpha \leq 1$. If $i \in I_{x}$, then

$$
\begin{aligned}
\left|u_{B\left(x_{i}, 3 r\right)}-u_{B(x, 9 R)}\right| \leq & \left|u_{B\left(x_{i}, 3 r\right)}-u_{B(x, 9 r)}\right| \\
& +\sum_{i=1}^{k}\left|u_{B\left(x, 2^{i} 9 r\right)}-u_{B\left(x, 2^{i-1} 9 r\right)}\right| \\
& +\left|u_{B\left(x, 2^{k} 9 r\right)}-u_{B(x, 9 R)}\right| .
\end{aligned}
$$

By the doubling condition and Poincaré inequality (3.4), we have

$$
\begin{aligned}
r^{\alpha}\left|u_{B\left(x_{i}, 3 r\right)}-u_{B(x, 9 r)}\right| & \leq C r^{\alpha} f_{B(x, 9 r)}\left|u-u_{B(x, 9 r)}\right| d \mu \\
& \leq C r^{s+\alpha}\left(f_{B(x, 18 r)} g^{t} d \mu\right)^{1 / t} \\
& \leq C R^{s+\alpha}\left(\mathcal{M} g^{t}(x)\right)^{1 / t},
\end{aligned}
$$

and, by the doubling condition, Poincare inequality (3.4), the fact that $r \leq 2^{i} 9 r$ for all $i$, and the selection of $k$,

$$
\begin{aligned}
r^{\alpha} \sum_{i=1}^{k}\left|u_{B\left(x, 2^{i} 9 r\right)}-u_{B\left(x, 2^{i-1} 9 r\right)}\right| & \leq C r^{\alpha} \sum_{i=1}^{k} f_{B\left(x, 2^{i} 9 r\right)}\left|u-u_{B\left(x, 2^{i} 9 r\right)}\right| d \mu \\
& \leq C \sum_{i=1}^{k}\left(2^{i} 9 r\right)^{s+\alpha}\left(f_{B\left(x, 2^{i+1} 9 r\right)} g^{t} d \mu\right)^{1 / t} \\
& \leq C R^{s+\alpha}\left(\mathcal{M} g^{t}(x)\right)^{1 / t} .
\end{aligned}
$$


Similarly we obtain that

$$
\begin{aligned}
r^{\alpha}\left|u_{B\left(x, 2^{k} 9 r\right)}-u_{B(x, 9 R)}\right| & \leq C R^{s+\alpha}\left(f_{B(x, 36 R)} g^{t} d \mu\right)^{1 / t} \\
& \leq C R^{s+\alpha}\left(\mathcal{M} g^{t}(x)\right)^{1 / t}
\end{aligned}
$$

If $i \in I_{y}$, we use balls $B\left(y, 2^{i} 9 r\right)$ instead of balls $B\left(x, 2^{i} 9 r\right)$ in (3.7). Estimates corresponding (3.8) and (3.9) are as above ( $x$ replaced by $y$ ) and, corresponding to (3.10),

$$
\begin{aligned}
r^{\alpha}\left|u_{B\left(y, 2^{k} 9 r\right)}-u_{B(x, 9 R)}\right| & \leq C R^{s+\alpha}\left(f_{B(x, 38 R)} g^{t} d \mu\right)^{1 / t} \\
& \leq C R^{s+\alpha}\left(\mathcal{M} g^{t}(x)\right)^{1 / t}
\end{aligned}
$$

Now, by (3.6)-(3.11) and the fact $R=d(x, y)$, we have

$$
r^{\alpha}\left|u_{B\left(x_{i}, 3 r\right)}-u_{B(x, 9 R)}\right| \leq C d(x, y)^{s+\alpha}\left(\left(\mathcal{M} g^{t}(x)\right)^{1 / t}+\left(\mathcal{M} g^{t}(y)\right)^{1 / t}\right) .
$$

If $s+\alpha>1$, then similar estimates as above show that if $i \in I_{x} \cup I_{y}$, then

$$
\begin{aligned}
r^{\alpha}\left|u_{B\left(x_{i}, 3 r\right)}-u_{B(x, 9 R)}\right| \leq C d(x, y) & \left(\mathcal{M}_{t(s+\alpha-1)} g^{t}(x)\right)^{1 / t} \\
& \left.+\left(\mathcal{M}_{t(s+\alpha-1)} g^{t}(y)\right)^{1 / t}\right) .
\end{aligned}
$$

These estimates together with (3.6) and the fact that there are bounded number of indices in $I_{x}$ and $I_{y}$ imply that Hajłasz gradient inequality (3.1) with desired exponent holds when $r<d(x, y)$.

Thus, we have proved the claim for $u_{r}^{\alpha}$. Since $\mathcal{M}_{\alpha}^{*} u \not \equiv \infty$, the claim for $\mathcal{M}_{\alpha}^{*} u$ follows from Remark 2.2 and Lemma 3.2.

Theorem 3.4. Let $s \geq 0, \alpha \geq 0$ and let $Q /(Q+s)<p<\infty$.

a) If $0<s+\alpha \leq 1$, there exists a constant $C>0$, such that

$$
\left\|\mathcal{M}_{\alpha}^{*} u\right\|_{\dot{M}^{s+\alpha, p}(X)} \leq C\|u\|_{\dot{M}^{s, p}(X)}
$$

for all $u \in \dot{M}^{s, p}(X)$ with $\mathcal{M}_{\alpha}^{*} u \neq \equiv \infty$.

b) If $1<s+\alpha \leq 1+Q / p$ and the measure lower bound condition holds, there exists a constant $C>0$ such that

$$
\left\|\mathcal{M}_{\alpha}^{*} u\right\|_{\dot{M}^{1, q}(X)} \leq C\|u\|_{\dot{M}^{s, p}(X)},
$$

where $q=Q p /(Q-(s+\alpha-1) p)$, for all $u \in \dot{M}^{s, p}(X)$ with $\mathcal{M}_{\alpha}^{*} u \neq \equiv \infty$. 
Proof: Let $Q /(Q+s) \leq t<p$. Let $g \in \mathcal{D}^{s}(u) \cap L^{p}(X)$.

a) If $0<s+\alpha \leq 1$, then by Theorem 3.3, the function $C\left(\mathcal{M} g^{t}\right)^{1 / t}$ is an $(s+\alpha)$-gradient of $\mathcal{M}_{\alpha}^{*} u$. Since $g \in L^{p}(X)$, the claim follows from the boundedness of the Hardy-Littlewood maximal operator in $L^{r}(X)$ for $r>1$.

b) If $1<s+\alpha \leq 1+Q / p$, then by Theorem 3.3, the function $\left(\mathcal{M}_{t(s+\alpha-1)} g^{t}\right)^{1 / t}$ is a 1 -gradient of $\mathcal{M}_{\alpha}^{*} u$. Since $g \in L^{p}(X)$, the claim follows from Theorem 2.1.

Remark 3.5. In the cases $s=1$ and $s=0$ of Theorem 3.4b), we obtain counterparts of the results [21, Theorem 2.1] and [21, Theorem 3.1] of Kinnunen and Saksman.

Remark 3.6. As a special case of Theorems 3.3 and 3.4 we obtain the following boundedness results for the discrete maximal operator $\mathcal{M}^{*}$ in $\dot{M}^{s, p}(X)$. Let $p>Q /(Q+s)$. Let $u \in \dot{M}^{s, p}(X)$ with $\mathcal{M}_{\alpha}^{*} u \not \equiv \infty$ and let $g \in \mathcal{D}^{s}(u) \cap L^{p}(X)$.

If $0<s \leq 1$, then $\tilde{g}=C\left(\mathcal{M} g^{t}\right)^{1 / t}$ is an $s$-Hajłasz gradient of $\mathcal{M}^{*} u$ for all $t \geq Q /(Q+s)$ and

$$
\left\|\mathcal{M}^{*} u\right\|_{\dot{M}^{s, p}(X)} \leq C\|u\|_{\dot{M}^{s, p}(X)} .
$$

If $1<s \leq 1+Q / p$, then $\tilde{g}=C\left(\mathcal{M}_{t(s-1)} g^{t}\right)^{1 / t}$ is a 1-Hajłasz gradient of $\mathcal{M}^{*} u$ for all $t \geq Q /(Q+s)$ and

$$
\left\|\mathcal{M}^{*} u\right\|_{\dot{M}^{1, q}(X)} \leq C\|u\|_{\dot{M}^{s, p}(X)},
$$

where $q=Q p /(Q-(s-1) p)$.

Moreover, when $s=1$, we obtain boundedness results for the discrete maximal operator $\mathcal{M}^{*}$ in (homogeneous) Hajłasz spaces $\dot{M}^{1, p}(X)$, proved earlier for $M^{1, p}(X)$ in $[\mathbf{1 9}]$ and $[\mathbf{2 2}]$.

\section{Hajłasz-Besov and Hajłasz-Triebel-Lizorkin spaces}

In this section, we study smoothing properties of the discrete fractional maximal operator in Besov and Triebel-Lizorkin spaces. For these spaces, we use a definition based on pointwise inequalities, introduced by Koskela, Yang, and Zhou in [25]. The constants $C$ of the theorems are independent of functions $u$, fractional gradients $\left(g_{k}\right)$, and of the construction of discrete fractional maximal function $\mathcal{M}_{\alpha}^{*} u$.

Let $u: X \rightarrow[-\infty, \infty]$ be a measurable function and let $0<s<$ $\infty$. Following [25], we say that a sequence of nonnegative measurable 
functions $\left(g_{k}\right)_{k \in \mathbb{Z}}$ is a fractional $s$-Hajłasz gradient of $u$ if there exists a set $E \subset X$ with $\mu(E)=0$ such that

$$
|u(x)-u(y)| \leq d(x, y)^{s}\left(g_{k}(x)+g_{k}(y)\right)
$$

for all $k \in \mathbb{Z}$ and all $x, y \in X \backslash E$ satisfying $2^{-k-1} \leq d(x, y)<2^{-k}$. The collection of all fractional $s$-Hajłasz gradients of $u$ is denoted by $\mathbb{D}^{s}(u)$.

For $0<p<\infty, 0<q \leq \infty$, and a sequence $\left(f_{k}\right)_{k \in \mathbb{Z}}$ of measurable functions, we write

$$
\left\|\left(f_{k}\right)_{k \in \mathbb{Z}}\right\|_{L^{p}\left(X, l^{q}\right)}=\|\|\left(f_{k}\right)_{k \in \mathbb{Z}}\left\|_{l^{q}}\right\|_{L^{p}(X)}
$$

and

$$
\left\|\left(f_{k}\right)_{k \in \mathbb{Z}}\right\|_{l^{q}\left(L^{p}(X)\right)}=\left\|\left(\left\|f_{k}\right\|_{L^{p}(X)}\right)_{k \in \mathbb{Z}}\right\|_{l^{q}},
$$

where $\left\|\left(f_{k}\right)\right\|_{l^{q}}=\left(\sum_{k \in \mathbb{Z}}\left|f_{k}\right|^{q}\right)^{1 / q}$ if $0<q<\infty$ and $\left\|\left(f_{k}\right)\right\|_{l^{\infty}}=\sup _{k \in \mathbb{Z}}\left|f_{k}\right|$.

The homogeneous Hajłasz-Triebel-Lizorkin space $\dot{M}_{p, q}^{s}(X)$ consists of measurable functions $u$ such that

$$
\|u\|_{\dot{M}_{p, q}^{s}(X)}=\inf _{\left(g_{k}\right) \in \mathbb{D}^{s}(u)}\left\|\left(g_{k}\right)\right\|_{L^{p}\left(X, l^{q}\right)}
$$

is finite. The Hajłasz-Triebel-Lizorkin space $M_{p, q}^{s}(X)$ is $\dot{M}_{p, q}^{s}(X) \cap$ $L^{p}(X)$ equipped with the norm

$$
\|u\|_{M_{p, q}^{s}(X)}=\|u\|_{L^{p}(X)}+\|u\|_{\dot{M}_{p, q}^{s}(X)} .
$$

The homogeneous Hajłasz-Besov space $\dot{N}_{p, q}^{s}(X)$ consists of measurable functions $u$ such that

$$
\|u\|_{\dot{N}_{p, q}^{s}(X)}=\inf _{\left(g_{k}\right) \in \mathbb{D}^{s}(u)}\left\|\left(g_{k}\right)\right\|_{l^{q}\left(L^{p}(X)\right)}
$$

is finite and the Hajłasz-Besov space $N_{p, q}^{s}(X)$ is $\dot{N}_{p, q}^{s}(X) \cap L^{p}(X)$ equipped with the norm

$$
\|u\|_{N_{p, q}^{s}(X)}=\|u\|_{L^{p}(X)}+\|u\|_{\dot{N}_{p, q}^{s}(X)} .
$$

Notice that $\dot{M}_{p, \infty}^{s}(X)$ is the homogeneous fractional Hajłasz space $\dot{M}^{s, p}(X)$, for the simple proof, see [25, Proposition 2.1]. The homogeneous Hajłasz-Triebel-Lizorkin space $\dot{M}_{p, q}^{s}\left(\mathbb{R}^{n}\right)$ coincides with the classical homogeneous Triebel-Lizorkin space $\dot{F}_{p, q}^{s}\left(\mathbb{R}^{n}\right)$ for $s \in(0,1), p \in$ $(n /(n+s), \infty)$ and $q \in(n /(n+s), \infty]$. Similarly, $\dot{N}_{p, q}^{s}\left(\mathbb{R}^{n}\right)$ coincides with the classical homogeneous Besov space $\dot{B}_{p, q}^{s}\left(\mathbb{R}^{n}\right)$ for $s \in(0,1)$, $p \in(n /(n+s), \infty)$, and $q \in(0, \infty]$ by [25, Theorem 1.2], see also [7, Chapters 1,3] about the different definitions of Besov and TriebelLizorkin spaces. For the definitions of $F_{p, q}^{s}\left(\mathbb{R}^{n}\right)$ and $B_{p, q}^{s}\left(\mathbb{R}^{n}\right)$, see $[\mathbf{3 0}]$. 
If $X$ supports a (weak) $(1, p)$-Poincaré inequality with $p \in(1, \infty)$, then for all $q \in(0, \infty)$, the spaces $\dot{M}_{p, q}^{1}(X)$ and $\dot{N}_{p, q}^{1}(X)$ are trivial, that is, they contain only constant functions, see [7, Theorem 4.1].

In the proofs, we will use a Poincaré type inequality from $[\mathbf{7}$, Lemma 2.1].

Lemma 4.1. Let $0<s<\infty$ and $0<p<Q / s$. Then for every $\varepsilon, \varepsilon^{\prime} \in(0, s)$ with $\varepsilon<\varepsilon^{\prime}$ there exists a constant $C>0$ such that for all measurable functions $u$ with $\left(g_{j}\right) \in \mathbb{D}^{s}(u), x \in X$, and $k \in \mathbb{Z}$,

$$
\begin{aligned}
& \inf _{c \in \mathbb{R}}\left(f_{B\left(x, 2^{-k}\right)}\right.\left.|u(y)-c|^{p^{*}(\varepsilon)} d \mu(y)\right)^{1 / p^{*}(\varepsilon)} \\
& \leq C 2^{-k \varepsilon^{\prime}} \sum_{j \geq k-2} 2^{-j\left(s-\varepsilon^{\prime}\right)}\left(f_{B\left(x, 2^{-k+1}\right)} g_{j}^{p} d \mu\right)^{1 / p},
\end{aligned}
$$

where $p^{*}(\varepsilon)=Q p /(Q-\varepsilon p)$.

If $u$ is locally integrable, $\left(g_{j}\right) \in \mathbb{D}^{s}(u)$ and $0<\varepsilon<\varepsilon^{\prime}<s<\infty$, then (4.1) with $p=Q /(Q+\varepsilon)$ and the Hölder inequality imply that for $t \geq Q /(Q+\varepsilon)$,

$$
\begin{aligned}
& f_{B\left(x, 2^{-k}\right)} \mid u- u_{B\left(x, 2^{-k}\right)} \mid d \mu \\
& \leq C 2^{-k \varepsilon^{\prime}} \sum_{j \geq k-2} 2^{-j\left(s-\varepsilon^{\prime}\right)}\left(f_{B\left(x, 2^{-k+1}\right)} g_{j}^{t} d \mu\right)^{1 / t} .
\end{aligned}
$$

Remark 4.2. If $Q /(Q+s) \leq p<\infty$, then $\dot{M}_{p, q}^{s}(X) \subset L_{\text {loc }}^{1}(X)$. Indeed, we have that $\dot{M}_{p, q}^{s}(X) \subset \dot{M}_{p, \infty}^{s}(X)=\dot{M}^{s, p}(X) \subset \dot{M}^{s, Q /(Q+s)}(X) \subset$ $L_{\text {loc }}^{1}(X)$, where the last inclusion follows from the discussion after Lemma 3.1. Similarly, if $Q /(Q+s)<p<\infty$, then $\dot{N}_{p, q}^{s}(X) \subset L_{\text {loc }}^{1}(X)$ by [7, Theorem 2.1(iv)].

An analogous proof as that of Lemma 3.2 gives us the following lemma.

Lemma 4.3. If $u_{i}, i \in \mathbb{N}$, are measurable functions with a common fractional $s$-Hajtasz gradient $\left(g_{k}\right)_{k \in \mathbb{Z}}$ and $u=\sup _{i} u_{i}$ is finite almost everywhere, then $\left(g_{k}\right)_{k \in \mathbb{Z}}$ is a fractional s-Hajtasz gradient of $u$.

We are now ready to state and prove our main results. Theorem 4.4 below gives a formula for an $(s+\alpha)$-Hajłasz gradient of $\mathcal{M}_{\alpha}^{*}$ in terms 
of an $s$-Hajłasz gradient of $u$. This easily implies the desired boundedness results for $\mathcal{M}_{\alpha}^{*}$ in homogeneous Hajłasz-Besov and Hajłasz-TriebelLizorkin spaces. For related results concerning Riesz potentials in the metric setting, see [32].

Theorem 4.4. Let $s>0$ and $\alpha \geq 0$ be such that $0<s+\alpha<1$. Let $0<\delta<1-s-\alpha, 0<\varepsilon<\varepsilon^{\prime}<s$, and $t \geq Q /(Q+\varepsilon)$. There is a constant $C>0$, such that if $u$ is locally integrable function with $\mathcal{M}_{\alpha}^{*} u \not \equiv \infty$ and if $\left(g_{k}\right) \in \mathbb{D}^{s}(u)$, then $\left(C \tilde{g}_{k}\right)$, where

$$
\tilde{g}_{k}=\sum_{j=-\infty}^{k} 2^{(j-k) \delta}\left(\mathcal{M} g_{j}^{t}\right)^{1 / t}+\sum_{j=k-7}^{\infty} 2^{(k-j)\left(s-\varepsilon^{\prime}\right)}\left(\mathcal{M} g_{j}^{t}\right)^{1 / t}
$$

is a fractional $(s+\alpha)$-Hajtasz gradient of $\mathcal{M}_{\alpha}^{*} u$.

Proof: Since $\mathcal{M}_{\alpha}^{*} u=\mathcal{M}_{\alpha}^{*}|u|$ and since every fractional $s$-Hajłasz gradient of $u$ is a fractional $s$-Hajłasz gradient of $|u|$, we may assume that $u \geq 0$.

Let $k \in \mathbb{Z}$ and let $x, y \in X$ such that $2^{-k-1} \leq d(x, y)<2^{-k}$. We will show that

$$
\left|u_{r}^{\alpha}(x)-u_{r}^{\alpha}(y)\right| \leq C d(x, y)^{s+\alpha}\left(\tilde{g}_{k}(x)+\tilde{g}_{k}(y)\right),
$$

where $C$ is independent of $r$ and $k$.

Assume first that $d(x, y)>r$. Then

$$
\begin{aligned}
\left|u_{r}(x)-u_{r}(y)\right| \leq & \left|u_{r}(x)-u_{B\left(x, 2^{-k+4}\right)}\right|+\left|u_{r}(y)-u_{B\left(x, 2^{-k+4}\right)}\right| \\
\leq & \sum_{i \in I_{x}} \varphi_{i}(x)\left|u_{B\left(x_{i}, 3 r\right)}-u_{B\left(x, 2^{-k+4}\right)}\right| \\
& +\sum_{i \in I_{y}} \varphi_{i}(y)\left|u_{B\left(x_{i}, 3 r\right)}-u_{B\left(x, 2^{-k+4}\right)}\right|
\end{aligned}
$$

where $I_{x}$ is a set of indices $i$ for which $x$ belongs to $B\left(x_{i}, 6 r\right)$ and $I_{y}$ the corresponding set for $y$. Let $m \in \mathbb{Z}$ be such that $2^{-m-1}<9 r \leq 2^{-m}$. Since $r<d(x, y)<2^{-k}$, it follows that $m \geq k-4$. If $i \in I_{x}$, we obtain

$$
\begin{aligned}
\left|u_{B\left(x_{i}, 3 r\right)}-u_{B\left(x, 2^{-k+4}\right)}\right| \leq & \left|u_{B\left(x_{i}, 3 r\right)}-u_{B\left(x, 2^{-m}\right)}\right| \\
& +\sum_{l=k-4}^{m-1}\left|u_{B\left(x, 2^{-l}\right)}-u_{B\left(x, 2^{-l-1}\right)}\right| \\
\leq & C \sum_{l=k-4}^{m} f_{B\left(x, 2^{-l}\right)}\left|u-u_{B\left(x, 2^{-l}\right)}\right| d \mu
\end{aligned}
$$


and hence Poincaré inequality (4.2) implies that

$$
\begin{aligned}
\left|u_{B\left(x_{i}, 3 r\right)}-u_{B\left(x, 2^{-k+4}\right)}\right| & \leq C \sum_{l=k-4}^{\infty} 2^{-l \varepsilon^{\prime}} \sum_{j=l-2}^{\infty} 2^{-j\left(s-\varepsilon^{\prime}\right)}\left(\mathcal{M} g_{j}^{t}(x)\right)^{1 / t} \\
& =C \sum_{j=k-6}^{\infty} 2^{-j\left(s-\varepsilon^{\prime}\right)}\left(\mathcal{M} g_{j}^{t}(x)\right)^{1 / t} \sum_{l=k-4}^{j+2} 2^{-l \varepsilon^{\prime}} \\
& \leq C 2^{-k \varepsilon^{\prime}} \sum_{j=k-6}^{\infty} 2^{-j\left(s-\varepsilon^{\prime}\right)}\left(\mathcal{M} g_{j}^{t}(x)\right)^{1 / t} \\
& =C 2^{-k s} \sum_{j=k-6}^{\infty} 2^{(k-j)\left(s-\varepsilon^{\prime}\right)}\left(\mathcal{M} g_{j}^{t}(x)\right)^{1 / t} \\
& \leq C 2^{-k s} \tilde{g}_{k}(x) .
\end{aligned}
$$

Similarly, if $i \in I_{y}$, then

$$
\begin{aligned}
\left|u_{B\left(x_{i}, 3 r\right)}-u_{B\left(x, 2^{-k+4}\right)}\right| \leq & \left|u_{B\left(x_{i}, 3 r\right)}-u_{B\left(y, 2^{-m}\right)}\right| \\
& +\sum_{l=k-5}^{m-1}\left|u_{B\left(y, 2^{-l}\right)}-u_{B\left(y, 2^{-l-1}\right)}\right| \\
& +\left|u_{B\left(y, 2^{-k+5}\right)}-u_{B\left(x, 2^{-k+4}\right)}\right| \\
\leq & C \sum_{l=k-5}^{m} f_{B\left(y, 2^{-l}\right)}\left|u-u_{B\left(y, 2^{-l}\right)}\right| d \mu,
\end{aligned}
$$

which implies that

$$
\left|u_{B\left(x_{i}, 3 r\right)}-u_{B\left(x, 2^{-k+4}\right)}\right| \leq C 2^{-k s} \tilde{g}_{k}(y) .
$$

It follows that

$$
\begin{aligned}
\left|u_{r}^{\alpha}(x)-u_{r}^{\alpha}(y)\right| & \leq C r^{\alpha} 2^{-k s}\left(\tilde{g}_{k}(x)+\tilde{g}_{k}(y)\right) \\
& \leq C d(x, y)^{s+\alpha}\left(\tilde{g}_{k}(x)+\tilde{g}_{k}(y)\right) .
\end{aligned}
$$

Suppose then that $d(x, y) \leq r$. Let $I_{x y}$ be a set of indices $i$ for which $x$ or $y$ belongs to $B\left(x_{i}, 6 r\right)$. Let $l$ be such that $2^{-l-1}<10 r \leq 2^{-l}$. Using the doubling condition, the properties of the functions $\varphi_{i}$, the fact that there are bounded number of indices in $I_{x y}$ and Poincaré inequality (4.2), 
we have that

$$
\begin{aligned}
\left|u_{r}^{\alpha}(x)-u_{r}^{\alpha}(y)\right| & \left.\leq r^{\alpha} \sum_{i=1}^{\infty} \mid \varphi_{i}(x)-\varphi_{i}(y)\right)|| u_{B\left(x_{i}, 3 r\right)}-u_{B\left(x, 2^{-l}\right)} \mid \\
& \leq C d(x, y) r^{\alpha-1} 2^{-l \varepsilon^{\prime}} \sum_{j=l-2}^{\infty} 2^{-j\left(s-\varepsilon^{\prime}\right)}\left(\mathcal{M} g_{j}^{t}(x)\right)^{1 / t} .
\end{aligned}
$$

Using the assumptions $0<\delta<1-\alpha-s, r \geq d(x, y)$, and $d(x, y)<2^{-k}$, we have that

$$
\begin{aligned}
d(x, y) r^{\alpha-1} 2^{-l \varepsilon^{\prime}} & \leq C d(x, y) r^{s+\alpha+\delta-1} 2^{l\left(s-\varepsilon^{\prime}+\delta\right)} \\
& \leq C d(x, y)^{s+\alpha+\delta} 2^{l\left(s-\varepsilon^{\prime}+\delta\right)} \\
& \leq C d(x, y)^{\alpha+s} 2^{(l-k) \delta+l\left(s-\varepsilon^{\prime}\right)}
\end{aligned}
$$

This together with (4.4) implies that

$$
\left|u_{r}^{\alpha}(x)-u_{r}^{\alpha}(y)\right| \leq C d(x, y)^{s+\alpha} \sum_{j=l-2}^{\infty} 2^{(l-k) \delta+(l-j)\left(s-\varepsilon^{\prime}\right)}\left(\mathcal{M} g_{j}^{t}(x)\right)^{1 / t} \text {. }
$$

By splitting the sum in two parts and using the estimates $l \leq j+2$ and $l \leq k$, we obtain

$$
\begin{aligned}
\sum_{j=l-2}^{\infty} 2^{(l-k) \delta+(l-j)\left(s-\varepsilon^{\prime}\right)}\left(\mathcal{M} g_{j}^{t}(x)\right)^{1 / t} & \\
= & \sum_{j=l-2}^{k-1} 2^{(l-k) \delta+(l-j)\left(s-\varepsilon^{\prime}\right)}\left(\mathcal{M} g_{j}^{t}(x)\right)^{1 / t} \\
& +\sum_{j=k}^{\infty} 2^{(l-k) \delta+(l-j)\left(s-\varepsilon^{\prime}\right)}\left(\mathcal{M} g_{j}^{t}(x)\right)^{1 / t} \\
\leq & C\left(\sum_{j=-\infty}^{k-1} 2^{(j-k) \delta}\left(\mathcal{M} g_{j}^{t}(x)\right)^{1 / t}+\sum_{j=k}^{\infty} 2^{(k-j)\left(s-\varepsilon^{\prime}\right)}\left(\mathcal{M} g_{j}^{t}(x)\right)^{1 / t}\right)
\end{aligned}
$$

which implies the claim for $u_{r}^{\alpha}$. The claim for $\mathcal{M}_{\alpha}^{*} u$ follows by Remark 2.2 and Lemma 4.3.

Theorem 4.5. Let $s>0$ and $\alpha \geq 0$ be such that $0<s+\alpha<1$. Let $Q /(Q+s)<p, q<\infty$. There exists a constant $C>0$ such that

$$
\left\|\mathcal{M}_{\alpha}^{*} u\right\|_{\dot{M}_{p, q}^{s+\alpha}(X)} \leq C\|u\|_{\dot{M}_{p, q}^{s}(X)}
$$

for all $u \in \dot{M}_{p, q}^{s}(X)$ with $\mathcal{M}_{\alpha}^{*} u \not \equiv \infty$. 
Proof: Let $u \in \dot{M}_{p, q}^{s}(X)$ and let $\left(g_{k}\right) \in \mathbb{D}^{s}(u)$ be such that $\left\|\left(g_{k}\right)\right\|_{L^{p}\left(X, l^{q}\right)} \leq$ $2\|u\|_{\dot{M}_{p, q}^{s}(X)}$. By Remark $4.2 u$ is locally integrable.

Let $\delta=\frac{1}{2}(1-(s+\alpha)), \varepsilon=\frac{1}{2} \max \left\{s, s+\frac{Q-Q r}{r}\right\}$, and $\varepsilon^{\prime}=\frac{1}{2}(\varepsilon+s)$, where $r=\min \{p, q\}$, and let $t=Q /(Q+\varepsilon)$. Then $0<\varepsilon<\varepsilon^{\prime}<s$ and $Q /(Q+s)<t<\min \{p, q\}$. By Theorem $4.4,\left(C \tilde{g}_{k}\right)$ defined by $(4.3)$ is a fractional $(s+\alpha)$-Hajłasz gradient of $\mathcal{M}_{\alpha}^{*} u$.

By the selection of $\left(g_{k}\right)$, it suffices to show that $\left(\tilde{g}_{k}\right) \in L^{p}\left(X, l^{q}\right)$ with $\left\|\left(\tilde{g}_{k}\right)_{k \in \mathbb{Z}}\right\|_{L^{p}\left(X, l^{q}\right)} \leq C\left\|\left(g_{k}\right)_{k \in \mathbb{Z}}\right\|_{L^{p}\left(X, l^{q}\right)}$. We estimate the $L^{p}\left(X, l^{q}\right)$ norm of

$$
\left(\sum_{j=-\infty}^{k} 2^{(j-k) \delta}\left(\mathcal{M} g_{j}^{t}\right)^{1 / t}\right)_{k \in \mathbb{Z}},
$$

the other part can be estimated similarly. If $q \geq 1$, we have, by the Hölder inequality, that

$$
\begin{aligned}
\sum_{k \in \mathbb{Z}}\left(\sum_{j=-\infty}^{k} 2^{(j-k) \delta}\left(\mathcal{M} g_{j}^{t}\right)^{1 / t}\right)^{q} & \leq C \sum_{k \in \mathbb{Z}} \sum_{j=-\infty}^{k} 2^{(j-k) \delta}\left(\mathcal{M} g_{j}^{t}\right)^{q / t} \\
& \leq C \sum_{j \in \mathbb{Z}}\left(\mathcal{M} g_{j}^{t}\right)^{q / t} \sum_{k=j}^{\infty} 2^{(j-k) \delta} \\
& \leq C \sum_{j \in \mathbb{Z}}\left(\mathcal{M} g_{j}^{t}\right)^{q / t}
\end{aligned}
$$

If $0<q<1$, we obtain the same estimate by using the elementary inequality $\left(\sum_{j} a_{j}\right)^{q} \leq \sum_{j} a_{j}^{q}$ for $a_{j} \geq 0$.

By the Fefferman-Stein vector valued maximal function theorem from [5] (for a metric space version, see for example $[\mathbf{2 8}]$ or $[\mathbf{8}]$ ), we obtain now the desired estimate

$$
\begin{aligned}
\left\|\left(\sum_{j=-\infty}^{k} 2^{(j-k) \delta}\left(\mathcal{M} g_{j}^{t}\right)^{1 / t}\right)_{k \in \mathbb{Z}}\right\|_{L^{p}\left(X, l^{q}\right)} & \leq C\left\|\left(\mathcal{M} g_{k}^{t}\right)_{k \in \mathbb{Z}}\right\|_{L^{p / t}\left(X, l^{q / t}\right)}^{1 / t} \\
& \leq C\left\|\left(g_{k}^{t}\right)_{k \in \mathbb{Z}}\right\|_{L^{p / t}\left(X, l^{q / t}\right)}^{1 / t} \\
& =C\left\|\left(g_{k}\right)_{k \in \mathbb{Z}}\right\|_{L^{p}\left(X, l^{q}\right)} .
\end{aligned}
$$

Theorem 4.6. Let $s>0$ and $\alpha \geq 0$ be such that $0<s+\alpha<1$. Let $Q /(Q+s)<p<\infty$ and $0<q<\infty$. Then there exists a constant $C>0$ such that

$$
\left\|\mathcal{M}_{\alpha}^{*} u\right\|_{\dot{N}_{p, q}^{s+\alpha}(X)} \leq C\|u\|_{\dot{N}_{p, q}^{s}(X)}
$$

for all $u \in \dot{N}_{p, q}^{s}(X)$ with $\mathcal{M}_{\alpha}^{*} u \not \equiv \infty$. 
Proof: Let $u \in \dot{M}_{p, q}^{s}(X)$ and let $\left(g_{k}\right) \in \mathbb{D}^{s}(u)$ be such that $\left\|\left(g_{k}\right)\right\|_{l^{q}\left(L^{p}(X)\right)} \leq$ $2\|u\|_{\dot{N}_{p, q}^{s}(X)}$. By Remark $4.2 u$ is locally integrable.

Let $\delta=\frac{1}{2}(1-(s+\alpha)), \varepsilon=\frac{1}{2} \max \left\{s, s+\frac{Q-Q p}{p}\right\}, \varepsilon^{\prime}=\frac{1}{2}(\varepsilon+s)$, and let $t=Q /(Q+\varepsilon)$. Then $0<\varepsilon<\varepsilon^{\prime}<s$ and $Q /(Q+s)<$ $t<p$. By Theorem 4.4, $\left(C \tilde{g}_{k}\right)$ defined by (4.3) is a fractional $(s+\alpha)$ Hajłasz gradient of $\mathcal{M}_{\alpha}^{*} u$. It suffices to show that $\left\|\left(\tilde{g}_{k}\right)\right\|_{l^{q}\left(L^{p}(X)\right)} \leq$ $C\left\|\left(g_{k}\right)\right\|_{l^{q}\left(L^{p}(X)\right)}$. Again, we estimate the first part of $\left(\tilde{g}_{k}\right)$ only. The second part can be estimated similarly.

Assume first that $p \geq 1$. By the Minkowski inequality and the HardyLittlewood maximal theorem,

$$
\begin{aligned}
\left\|\sum_{j=-\infty}^{k} 2^{(j-k) \delta}\left(\mathcal{M} g_{j}^{t}\right)^{1 / t}\right\|_{L^{p}(X)} & \leq \sum_{j=-\infty}^{k} 2^{(j-k) \delta}\left\|\left(\mathcal{M} g_{j}^{t}\right)^{1 / t}\right\|_{L^{p}(X)} \\
& \leq \sum_{j=-\infty}^{k} 2^{(j-k) \delta}\left\|g_{j}\right\|_{L^{p}(X)} .
\end{aligned}
$$

If $q \geq 1$, we have by the Hölder inequality,

$$
\begin{aligned}
\sum_{k \in \mathbb{Z}}\left(\sum_{j=-\infty}^{k} 2^{(j-k) \delta}\left\|g_{j}\right\|_{L^{p}(X)}\right)^{q} & \leq C \sum_{k \in \mathbb{Z}} \sum_{j=-\infty}^{k} 2^{(j-k) \delta}\left\|g_{j}\right\|_{L^{p}(X)}^{q} \\
& \leq C \sum_{j \in \mathbb{Z}}\left\|g_{j}\right\|_{L^{p}(X)}^{q} \sum_{k=j}^{\infty} 2^{(j-k) \delta} \\
& \leq C \sum_{j \in \mathbb{Z}}\left\|g_{j}\right\|_{L^{p}(X)}^{q} .
\end{aligned}
$$

If $0<q<1$, we use the inequality $\left(\sum_{j} a_{j}\right)^{q} \leq \sum_{j} a_{j}^{q}$ for $a_{j} \geq 0$ instead of the Hölder inequality.

Assume then that $Q /(Q+s)<p<1$. Then the inequality $\left(\sum_{j} a_{j}\right)^{p} \leq$ $\sum_{j} a_{j}^{p}$ and the Hardy-Littlewood maximal theorem imply that

$$
\begin{aligned}
\left\|\sum_{j=-\infty}^{k} 2^{(j-k) \delta}\left(\mathcal{M} g_{j}^{t}\right)^{1 / t}\right\|_{L^{p}(X)}^{p} & \leq \sum_{j=-\infty}^{k} 2^{(j-k) \delta p}\left\|\left(\mathcal{M} g_{j}^{t}\right)^{1 / t}\right\|_{L^{p}(X)}^{p} \\
& \leq \sum_{j=-\infty}^{k} 2^{(j-k) \delta p}\left\|g_{j}\right\|_{L^{p}(X)}^{p} .
\end{aligned}
$$


If $q \geq p$, we have by the Hölder inequality,

$$
\begin{aligned}
\sum_{k \in \mathbb{Z}}\left\|\sum_{j=-\infty}^{k} 2^{(j-k) \delta}\left(\mathcal{M} g_{j}^{t}\right)^{1 / t}\right\|_{L^{p}(X)}^{q} & \leq \sum_{k \in \mathbb{Z}}\left(\sum_{j=-\infty}^{k} 2^{(j-k) \delta p}\left\|g_{j}\right\|_{L^{p}(X)}^{p}\right)^{q / p} \\
& \leq C \sum_{k \in \mathbb{Z}} \sum_{j=-\infty}^{k} 2^{(j-k) \delta p}\left\|g_{j}\right\|_{L^{p}(X)}^{q} \\
& \leq C \sum_{j \in \mathbb{Z}}\left\|g_{j}\right\|_{L^{p}(X)}^{q} \sum_{k=j}^{\infty} 2^{(j-k) \delta p} \\
& \leq C \sum_{j \in \mathbb{Z}}\left\|g_{j}\right\|_{L^{p}(X)}^{q} .
\end{aligned}
$$

If $0<q<p$, we use the inequality $\left(\sum_{j} a_{j}\right)^{q / p} \leq \sum_{j} a_{j}^{q / p}$ instead of the Hölder inequality.

Theorems 4.5, 4.6, and the Hardy-Littlewood maximal theorem imply the following results for the discrete maximal operator.

Theorem 4.7. Let $0<s<1$.

a) If $Q /(Q+s)<p, q<\infty$, then there exists a constant $C>0$ such that

$$
\left\|\mathcal{M}^{*} u\right\|_{\dot{M}_{p, q}^{s}(X)} \leq C\|u\|_{\dot{M}_{p, q}^{s}(X)},
$$

whenever $u \in \dot{M}_{p, q}^{s}(X)$ and $\mathcal{M}^{*} u \not \equiv \infty$.

b) If $1<p, q<\infty$, then there exists a constant $C>0$ such that

$$
\left\|\mathcal{M}^{*} u\right\|_{M_{p, q}^{s}(X)} \leq C\|u\|_{M_{p, q}^{s}(X)},
$$

for all $u \in M_{p, q}^{s}(X)$.

Theorem 4.8. Let $0<s<1$.

a) If $Q /(Q+s)<p<\infty$ and $0<q<\infty$, there exists a constant $C>$ 0 such that

$$
\left\|\mathcal{M}^{*} u\right\|_{\dot{N}_{p, q}^{s}(X)} \leq C\|u\|_{\dot{N}_{p, q}^{s}(X)}
$$

for all $u \in \dot{N}_{p, q}^{s}(X)$ with $\mathcal{M}^{*} u \neq \equiv \infty$.

b) If $1<p<\infty$ and $0<q<\infty$, there exists a constant $C>0$ such that

$$
\left\|\mathcal{M}^{*} u\right\|_{N_{p, q}^{s}(X)} \leq C\|u\|_{N_{p, q}^{s}(X)}
$$

for all $u \in N_{p, q}^{s}(X)$. 
Acknowledgements. The research was supported by the Academy of Finland, grants no. 135561 and 141021. The authors want to thank the referee for helpful comments.

\section{References}

[1] D. Aalto and J. Kinnunen, The discrete maximal operator in metric spaces, J. Anal. Math. 111 (2010), 369-390. DOI: 10.1007/s11854-010-0022-3.

[2] J. M. Aldaz and J. PÉrez LÁzaro, Functions of bounded variation, the derivative of the one dimensional maximal function, and applications to inequalities, Trans. Amer. Math. Soc. 359(5) (2007), 2443-2461 (electronic). DOI: 10.1090/S0002-9947-06-04347 $-9$.

[3] S. M. BuckLey, Is the maximal function of a Lipschitz function continuous?, Ann. Acad. Sci. Fenn. Math. 24(2) (1999), 519-528.

[4] D. E. Edmunds, V. Kokilashvili, and A. Meskhi, "Bounded and compact integral operators", Mathematics and its Applications 543, Kluwer Academic Publishers, Dordrecht, 2002. DOI: 10.1007/978-94-015-9922-1.

[5] C. Fefferman and E. M. Stein, Some maximal inequalities, Amer. J. Math. 93 (1971), 107-115. DOI: 10.2307/2373450.

[6] I. Genebashvili, A. Gogatishvili, V. Kokilashvili, and M. KRBEC, "Weight theory for integral transforms on spaces of homogeneous type", Pitman Monographs and Surveys in Pure and Applied Mathematics 92, Longman, Harlow, 1998.

[7] A. Gogatishvili, P. Koskela, And Y. Zhou, Characterizations of Besov and Triebel-Lizorkin spaces on metric measure spaces, Forum Math. 25(4) (2013), 787-819. DOI: 10.1515/form.2011.135.

[8] L. Grafakos, L. LiU, And D. Yang, Vector-valued singular integrals and maximal functions on spaces of homogeneous type, Math. Scand. 104(2) (2009), 296-310.

[9] P. HajŁasz, Sobolev spaces on an arbitrary metric space, Potential Anal. 5(4) (1996), 403-415. DOI: 10.1007/BF00275475.

[10] P. HajŁasz, Sobolev spaces on metric-measure spaces, in: "Heat kernels and analysis on manifolds, graphs, and metric spaces", Contemp. Math. 338, Amer. Math. Soc., Providence, RI, 2003, pp. 173-218. DOI: 10.1090/conm/338/06074.

[11] P. HajŁasz and P. Koskela, Sobolev met Poincaré, Mem. Amer. Math. Soc. 145(688) (2000), 101 pp. DOI: 10.1090/memo/0688. 
[12] P. HajŁasz and J. MalÝ, On approximate differentiability of the maximal function, Proc. Amer. Math. Soc. 138(1) (2010), 165-174. DOI : $10.1090 /$ S0002-9939-09-09971-7.

[13] P. HajŁasz and J. Onninen, On boundedness of maximal functions in Sobolev spaces, Ann. Acad. Sci. Fenn. Math. 29(1) (2004), $167-176$.

[14] T. Heikkinen, J. Kinnunen, J. Nuutinen, and H. Tuominen, Mapping properties of the discrete fractional maximal operator in metric measure spaces, Kyoto J. Math. 53(3) (2013), 693-712. DOI: 10.1215/21562261-2265932.

[15] T. Heikkinen, P. Koskela, And H. Tuominen, Sobolev-type spaces from generalized Poincaré inequalities, Studia Math. 181(1) (2007), 1-16. DOI: 10.4064/sm181-1-1.

[16] T. Heikkinen, J. Lehrbäck, J. Nuutinen, And H. TuomiNEN, Fractional maximal functions in metric measure spaces, Anal. Geom. Metr. Spaces 1 (2013), 147-162. DOI: 10.2478/agms-20130002.

[17] J. Hu, A note on Hajłasz-Sobolev spaces on fractals, J. Math. Anal. Appl. 280(1) (2003), 91-101. DOI : 10.1016/S0022-247X (03)00039-8.

[18] J. Kinnunen, The Hardy-Littlewood maximal function of a Sobolev function, Israel J. Math. 100 (1997), 117-124. DOI: $10.1007 / B F 02773636$.

[19] J. Kinnunen And V. Latvala, Lebesgue points for Sobolev functions on metric spaces, Rev. Mat. Iberoamericana 18(3) (2002), 685-700. DOI : 10.4171/RMI/332.

[20] J. Kinnunen And P. Lindqvist, The derivative of the maximal function, J. Reine Angew. Math. 503 (1998), 161-167.

[21] J. Kinnunen And E. Saksman, Regularity of the fractional maximal function, Bull. London Math. Soc. 35(4) (2003), 529-535. DOI: 10.1112/S0024609303002017.

[22] J. Kinnunen And H. Tuominen, Pointwise behaviour of $M^{1,1}$ Sobolev functions, Math. Z. 257(3) (2007), 613-630. DOI : 10.1007/ s00209-007-0139-y.

[23] S. KorRY, Boundedness of Hardy-Littlewood maximal operator in the framework of Lizorkin-Triebel spaces, Rev. Mat. Complut. 15(2) (2002), 401-416.

[24] S. KorRy, A class of bounded operators on Sobolev spaces, Arch. Math. (Basel) 82(1) (2004), 40-50. DOI: 10.1007/s00013-0030416-x.

[25] P. Koskela, D. YAng, And Y. Zhou, Pointwise characterizations of Besov and Triebel-Lizorkin spaces and quasiconformal mappings, 
Adv. Math. 226(4) (2011), 3579-3621. DOI: 10.1016/j.aim. 2010. 10.020 .

[26] H. Luiro, Continuity of the maximal operator in Sobolev spaces, Proc. Amer. Math. Soc. 135(1) (2007), 243-251 (electronic). DOI: 10.1090/S0002-9939-06-08455-3.

[27] H. Luiro, On the regularity of the Hardy-Littlewood maximal operator on subdomains of $\mathbb{R}^{n}$, Proc. Edinb. Math. Soc. (2) 53(1) (2010), 211-237. DOI: 10.1017/S0013091507000867.

[28] Y. Sawano, Sharp estimates of the modified Hardy-Littlewood maximal operator on the nonhomogeneous space via covering lemmas, Hokkaido Math. J. 34(2) (2005), 435-458. DOI: 10.14492/hokmj/1285766231.

[29] H. TANAKA, A remark on the derivative of the one-dimensional Hardy-Littlewood maximal function, Bull. Austral. Math. Soc. 65(2) (2002), 253-258. DOI : 10.1017/S0004972700020293.

[30] H. TRIEBel, "Theory of function spaces", Monographs in Mathematics 78, Birkhäuser Verlag, Basel, 1983. DOI: 10.1007/978-30346-0416-1.

[31] D. YANG, New characterizations of Hajłasz-Sobolev spaces on metric spaces, Sci. China Ser. A 46(5) (2003), 675-689. DOI: 10.1360/02ys0343.

[32] D. YANG, Riesz potentials in Besov and Triebel-Lizorkin spaces over spaces of homogeneous type, Potential Anal. 19(2) (2003), 193-210. DOI: 10.1023/A:1023217617339.

Toni Heikkinen:

Department of Mathematics

P. O. Box 11100

FI-00076 Aalto University

Finland

E-mail address: toni.heikkinen@aalto.fi

Heli Tuominen:

Department of Mathematics and Statistics

P. O. Box 35

FI-40014 University of Jyväskylä

Finland

E-mail address: heli.m.tuominen@jyu.fi

Primera versió rebuda el 2 d'abril de 2013, darrera versió rebuda el 20 de desembre de 2013. 